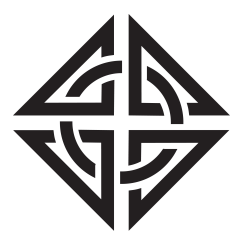

SCIENTIA

I RAN I C A
Sharif University of Technology

Scientia Iranica

Transactions E: Industrial Engineering

http://scientiairanica.sharif.edu

\title{
Reliability and cost optimization of a system with k-out-of-n configuration and choice of decreasing the components failure rates
}

\author{
M. Sharifi, ${ }^{a, *}$ Gh. Cheragh ${ }^{a}$, K. Dashti Maljaii ${ }^{a}$, A. Zaretalab ${ }^{b}$, and M. Shahriaric \\ a. Faculty of Industrial \& Mechanical Engineering, Qazvin Branch, Islamic Azad University, Qazvin, Iran. \\ b. Department of Industrial Engineering, Amirkabir University of Technology, 424 Hafez Ave., Tehran, Iran. \\ c. Faculty of Management and Accounting, South Tehran Branch, Islamic Azad University, Tehran, Iran.
}

Received 5 March 2019; received in revised form 25 December 2019; accepted 1 June 2020

\author{
KEYWORDS \\ Reliability; \\ Redundancy \\ allocation problem; \\ NSGA-II; \\ NRGA; \\ Response surface \\ methodology.
}

\begin{abstract}
This paper presents a new redundancy allocation problem for a system with the k-out-of-n configuration at the subsystems level with two active and cold standby redundancy strategies. The failure rate of components in each subsystem depends on the number of working components. The components are non-reparable, and the failure rate of the component can be decreased through some preventive maintenance actions. The model has two objective functions: maximizing the system reliability and minimizing the system costs. The system aims to find the type and number of components in each subsystem, redundancy strategy of subsystems, as well as the decreased values of components failure rates in subsystems. Since the redundancy allocation problem belongs to NP-hard problems, two metaheuristic algorithms including Non-dominated Sorting Genetic Algorithm II (NSGA-II) and Non-dominated Ranked Genetic Algorithm (NRGA) were used to solve the presented model. To tune algorithms parameters, we used response surface methodology. Besides, these algorithms were compared using five different performance metrics. Finally, the hypothesis test was used to analyze the results of the algorithms.
\end{abstract}

(C) 2021 Sharif University of Technology. All rights reserved.

\section{Introduction}

Currently, many studies have been conducted in the field of reliability to achieve more reliable systems. The Redundancy Allocation Problem (RAP) is the most important in this area. RAP aims to maximize the

\footnotetext{
*. Corresponding author.

E-mail addresses: m.sharifi@qiau.ac.ir (M. Sharifi);

ghasemcheragh@yahoo.com (Gh. Cheragh);

kamidashti@yahoo.com (K. Dashti Maljaii);

arash_zaretalab@yahoo.com (A. Zaretalab);

shahriari.mr@gmail.com (M. Shahriari)
}

doi: $10.24200 /$ sci.2020.52944.2960 system reliability by increasing redundant components of subsystems under some constraints. This problem was first presented by Fyffe et al. [1] and was solved by dynamic programming. Chern [2] proved that RAP belongs to the NP-hard problem when the number of subsystems increases. Therefore, many heuristic and meta-heuristic methods have been used to solve this problem.

There are much real-world manufacturing and operational systems that increase their reliability through the concepts of RAP, which can be counted, including aircraft engines, the number of pumps at a water pumping station, and so on. Considering the nature of these manufacturing and operating systems, many hy- 
potheses and limitations have been added to the RAP to draw the problem closer to real-world conditions.

Therefore, researchers categorized this problem from different aspects, including categorization based on the functional status of the components (binary or multi-state), the type of component failure rate (constant or time-dependent), components configuration in the system (active or standby).

Considering the importance of the system reliability and system cost, in many studies, both objectives are considered as objective functions, and this problem is transformed into a two-objective problem (and even more than two).

In this paper, we investigate a Multi-Objective RAP (MORAP) whose failure rates depend on the number of working elements. The subsystems are kout-of-n, and the failure rate of components can be reduced with spending money. The objective functions of the model are maximizing system reliability and minimizing system weight. The type of each subsystem component and subsystem redundancy strategies are the system variables. Since the model is NP-hard, we used Non-dominated Sorting Genetic Algorithm II (NSGA-II) and Non-dominated Ranked Genetic Algorithm (NRGA) for solving this model.

This paper is organized as follows: In Section 2, we present a literature review to confirm that there exist no studies that exactly meet these research conditions. In Section 3, we discussed the mathematical model and system assumptions. In Section 4, the NSGA-II and NRGA algorithms are presented. In Section 5, a numerical example is presented to compare the algorithms results. Section 6 is the managerial insights, and the final section deals with the conclusion and further studies.

\section{Literature review}

In real-world problems, many parameters affect the system reliability. One of the most important factors is the failure rate of the components. This parameter in RAP studies has two categories: Constant Failure Rate (CFR) and time-dependent failure rate.

Regarding CFR models, Misra and Sharma [3] presented a RAP model with the choice of allocating identical components to each subsystem and active redundancy strategy, then solved the presented model with zero-one programming. Ida [4] used a genetic algorithm to solve RAP with multi-failure components. Coit and Smith [5] presented a RAP model with the choice of allocating non-identical elements to each subsystem and solved the presented model using dynamic programming, integer programming, mixed non-linear integer programming, and compared the results with those of genetic algorithm. Coit and Liu [6] were the first who worked on a system with k-out-of-n sub-systems. They predefined active or cold standby redundancy strategies for each subsystem and solved the problem using integer programming. Hsieh and You [7] presented a new two-stage method based on the immune algorithm to solve the RAP under nonlinear weight, volume, and cost constraints. Hsieh and Yeh [8] used penalty-guided bees search to solve RAP.

All presented studies have been conducted on single-objective models. Busacca et al. [9] presented a two-objective model. The model objectives were maximizing the system reliability and net profit and used a multi-objective genetic algorithm to solve the problem. Coit and Jin [10] worked on a MORAP with maximizing system reliability and minimizing the variance of results. Baharanwala et al. [11] used NSGA to solve the MORAP. The objectives of the model are maximizing system reliability, minimizing the system cost, weight, and variance of reliability. In the presented model, each subsystem has a lower and upper limit for components allocation. Salazar et al. [12] solved three different RAP models using NSGAII. The objective functions of the models are maximizing system reliability and minimizing the system cost. Kulturel-Konak [13] worked on a MORAP model using the Tabu search algorithm. Taboada et al. [14] presented two methods for decreasing the size of Pareto solutions and used the presented method for solving MORAP. Taboada and Coit [15] presented an MOEA evolutionary algorithm to solve the MORAP with a new crossover operator that increased the variations of the solutions. Liang and Lo [16] presented a variable neighborhood search for solving MORAP and solved three different models and compared the results with NSGA-II results. Soylu and Ulusoy [17] worked on MORAP and contributed Pareto solutions to smalland large-scale problems. Then they classified the solutions using the UTADIS method.

Concerning time-dependent failure rate models, Coit [18] presented a new RAP with a switching system. Also, he used the K-Erlang distribution function for components and solved the problem using dynamic programming. Later on, Coit [19] considered two different active and cold standby redundancy strategies for each subsystem. Tavakkoli-Moghaddam et al. [20], Safari and Tavakkoli-Moghaddam [21] solved that problem using a genetic algorithm in 2008 and a memetic algorithm in 2010. Amari [22] presented an enumeration method for evaluating the reliability of $k$ out-of-n systems with a cold standby redundancy strategy. Dhingra [23] used mixed goal programming and goal attainment to produce Pareto optimal solutions in a fourth-level system to maximizing reliability and minimizing system costs and weight. Ghafarian Salehi Nezhad et al. [24] presented a four-phase algorithm to improve reliability in series-parallel systems with redundancy allocation. They combined an Ant Colony 
Optimization (ACO) algorithm as a meta-heuristic phase, and three other heuristics to develop a solving methodology for RAP. Azaron et al. [25] used the shortest path method in stochastic graphs to evaluate the reliability of a cold standby system with non-identical elements. Azaron et al. [26] salved a multi-state cold standby RAP and non-repairable components with the GADSCRRSU method. Ebrahim Nezhad et al. [27] presented a new method for MORAP with the choice of allocating identical elements and predefined active and cold standby redundancy strategies with maximizing reliability and net profit objective functions. Chambari et al. [28] used multi-objective particle swarm optimization and NSGA-II for a MORAP with the choice of selecting the redundancy strategy of each subsystem. Azimi et al. [29] Solved a RAP with kout-of-n configuration and non-exponential repairable components. They used optimization via simulation technique to solve the presented RAP. Pourkarim Guilani et al. [30] worked on a bi-objective reliability model with three-state components. They used multiobjective Strength Pareto Evolutionary Algorithm II (SPEA-II) and NSGA-II to solve the presented model.

In all mentioned research studies, the failure rate of working elements is fixed. For example, for a system with one working component, the failure rate of this component is equal to the failure rates of components in a system with 10 working components. Sharifi et al. [31] presented a formula for evaluating the reliability of a k-out-of-n system when the components failure rates depend on the number of working elements. In the presented k-out-of-n system, when a component failed, the failure rates of the remaining components increases.

Table 1 contains several recent studies on the reliability field, along with a summary of the model behaviors.

\section{Mathematical model}

In this paper, the system has $s$ k-out-of-n sub-systems, and its objectives are maximizing system reliability and minimizing system weight under two linear constraints.

\subsection{Model assumptions}

The main assumptions of the current paper are as follows:

- The system has $s$ subsystems

- Each subsystem is k-out-of-n

- Subsystem components are identical

- Components are binary states

- The probability of working switching system for cold standby subsystems is equal to $p$

- The failure rate of working components depends on the number of working components
- The components are non-repairable, and

- The system parameters are constant

The notations which are used in this paper are as follows:

$s \quad$ Number of subsystems

$i \quad$ Subsystem index

$k_{i} \quad$ Minimum necessary components in $i$ th subsystem

$n_{i} \quad$ Number of components in $i$ th subsystem

$n \quad$ The set of $n_{i}\left(n_{1}, \ldots, n_{s}\right)$

$n_{\max , i} \quad$ The upper limit of $n_{i}\left(n_{i} \leq n_{\max , i} ; i=\right.$ $1, \ldots, s)$

$m_{i} \quad$ Number of available component types in $i$ th subsystem $(i=1, \ldots, s)$

$z_{i} \quad$ Component type index in $i$ th subsystem $z_{i} \in\left(1, \ldots, m_{i}\right)$

$z \quad$ Set of $z_{i} \in\left(1, \ldots, m_{i}\right)$

$t \quad$ Mission time

$\lambda_{i z_{i} k_{i}} \quad$ The failure rate of type $z_{i}$ component in $i$ th subsystem when the subsystem working with $k$ components

$\lambda_{i z_{i} k_{i}}^{\prime} \quad$ The reduced failure rate of type $z_{i}$ component in $i$ th subsystem when the subsystem working with $k$ components

$w_{i z_{i}} \quad$ Weight of component, type $z_{i}$ in $i$ th subsystem

$c_{i z_{i}} \quad$ Cost of component, type $z_{i}$ in $i$ th subsystem

$c_{i z_{i}}^{\prime} \quad$ Cost of reducing the failure rate of each component, type $z_{i}$ in $i$ th subsystem

$\theta_{i z_{i}} \quad$ Cost parameter of internal relation for component, type $z_{i}$ in $i$ th subsystem

$\beta_{i z_{i}} \quad$ Reducing factor of failure rate for type $z_{i}$ component in $i$ th subsystem

$W \quad$ Total acceptable weight of the system

$A \quad$ Index of subsystems with active redundancy strategy

$S \quad$ Index of subsystems with cold standby redundancy strategy

$R_{l_{1}} \quad$ Reliability of the subsystem with active redundancy strategy

$R_{l_{2}} \quad$ Reliability of the subsystem with cold standby redundancy strategy

$R_{l} \quad$ System reliability

$P \quad$ Probability of working the switch in switching time

\subsection{Mathematical model}

In the presented mathematical model, the reliability 


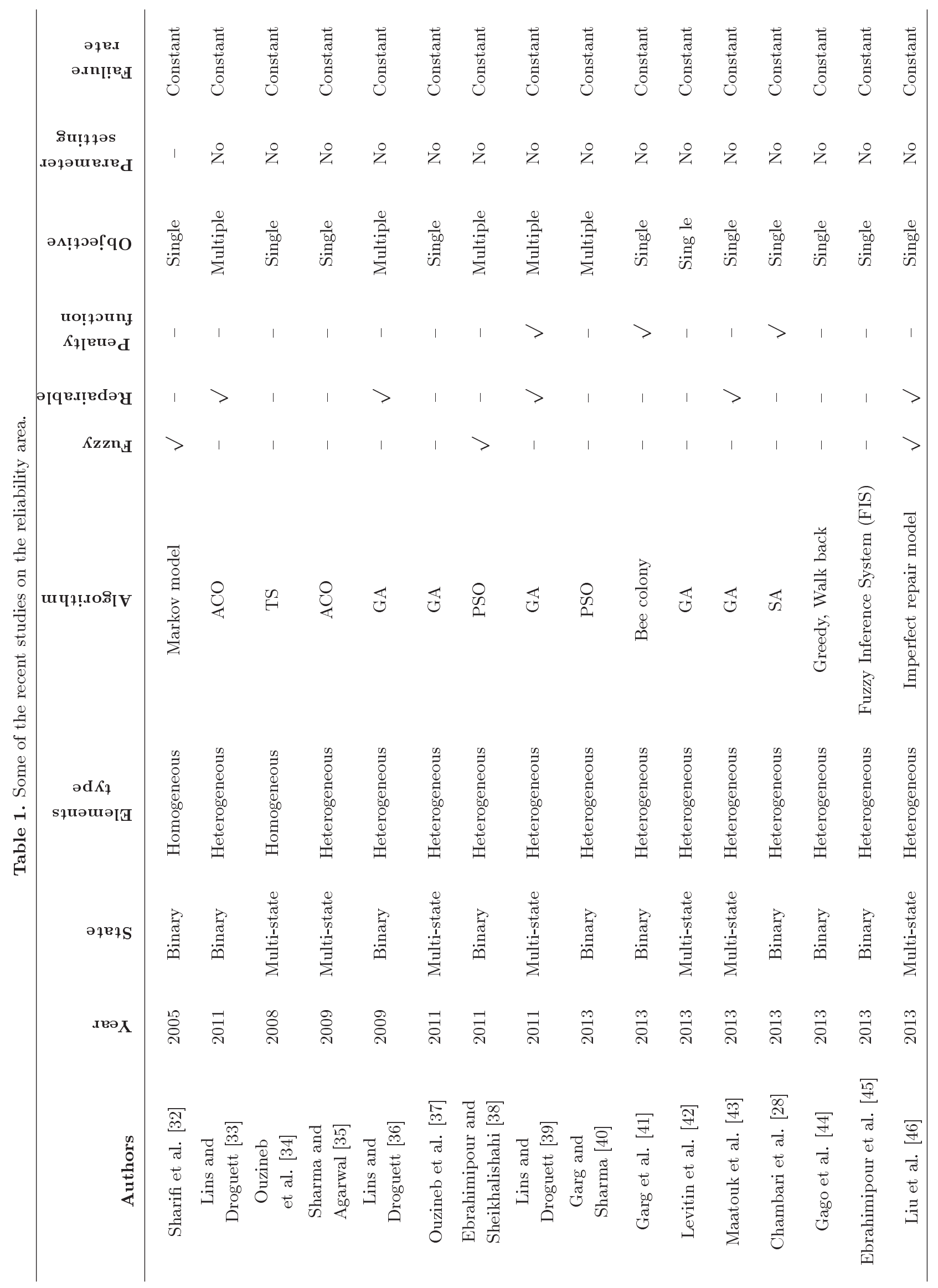




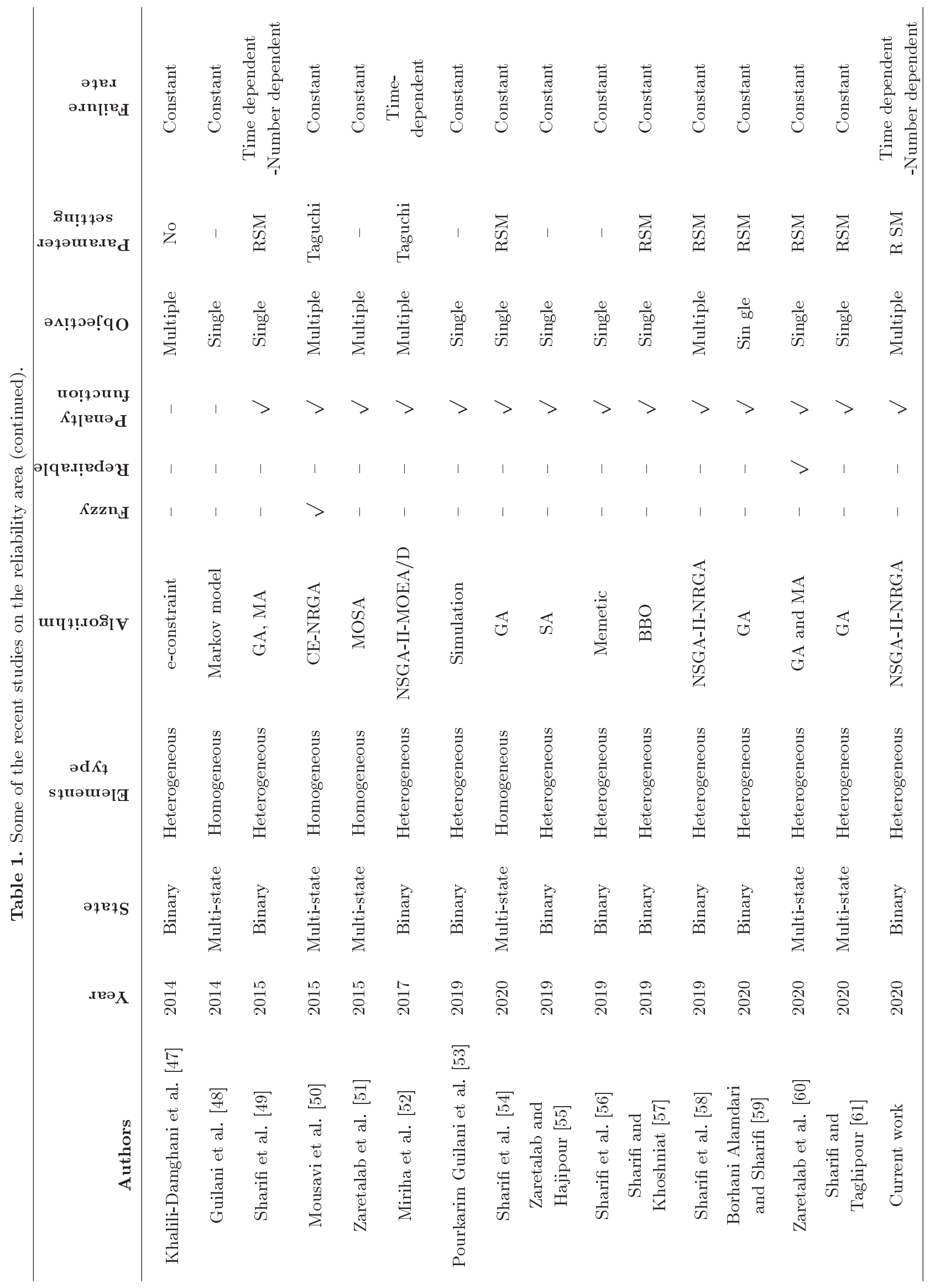


and cost of the system are designed to be optimized simultaneously. One of the most important system constraints in the RAPs is the system weight constraint. This constraint is considered in the proposed mathematical model. Another considered constraint is the upper bound for reducing components failure rates. In light of these explanations, the decision variables of this problem are the number of components in each subsystem and reducing the factor of failure rate for all of the components in each subsystem.

$$
\begin{aligned}
& \max R_{l}(t)=\left\{\prod_{i \in A} R_{l_{1}}(t)\right\} \times\left\{\prod_{i \in S} R_{l_{2}}(t)\right\}, \\
& \min C=\sum_{i=1}^{s}\left\{c_{i z_{i}}\left\{n_{i}+f\left(\theta_{i z_{i}}\right)\right\}+c_{i z_{i}}^{\prime} f\left(\beta_{i}\right)\right\},
\end{aligned}
$$

s.t.:

$$
\begin{aligned}
& \sum_{i=1}^{s} w_{i z_{i}} n_{i} \leq W, \\
& 0 \leq \beta_{i} \leq a_{i}, \\
& n_{i} \in\left(k_{i}, 2, \ldots, n_{\max }\right) ; \quad i=(1,2, \ldots, s), \\
& z_{i} \in\left(1,2, \ldots, m_{i}\right) ; \quad i=(1,2, \ldots, s) .
\end{aligned}
$$

In this model, Eq. (1) is the first objective function, i.e., maximizing the system reliability. Eq. (2) is the second objective function, i.e., minimizing the system cost. We consider that the system cost contains the cost of redundant components, the cost of internal relation [62], and the cost of reducing the components failure rates. In this paper, $f\left(\beta_{i}\right)$ and $f\left(\theta_{i z_{i}}\right)$ are defined as follows:

$$
f\left(\beta_{i}\right)=e^{\beta_{i} \lambda_{i z_{i}}}, \quad f\left(\theta_{i z_{i}}\right)=e^{\theta_{i z_{i}} n_{i}} .
$$

Eq. (3) is the constraint of system weight. Eq. (4) is the upper and lower limits of reducing components failure rate and Eqs. (5) and (6) define the maximum number and type of components in each subsystem, respectively. The first objective function is divided into two parts. The first part is the reliability of the subsystems with an active redundancy strategy, and the second part is the reliability of the subsystems with a cold standby redundancy strategy.

The reliability of a system with $n$ identical component and active redundancy strategy when the failure rate of the component is related to the number of the working component can be calculated as follows [31]:

$$
\begin{aligned}
R(t)= & \left(\prod_{j=k}^{n} \lambda_{j}\right) \\
& \times \sum_{i=k}^{n}\left\{\frac{n !}{i(k-1) !}\left(\prod_{\substack{\theta=k \\
\theta \neq i}}^{n} \frac{1}{\theta \times \lambda_{\theta}-i \times \lambda_{i}}\right)\right. \\
& \left.\times \frac{e^{-i \times \lambda_{i} \times t}}{\lambda_{i}}\right\} .
\end{aligned}
$$

In Eq. (7), $\lambda_{i}$ is the failure rate of components when the system is working with $i$ components. In a real situation, when a component fails, the load on other working components increases. Eq. (9) makes a relation between the failure rates of working components [31]:

$$
\lambda_{k}=\frac{k-\gamma(k-1)}{k} \lambda_{1}
$$

In Eq. (9), $0 \leq \gamma \leq 1$ can tune the relations between failure rates of the component. When $\gamma=0$ the failure rate of working components is independent of the number of working components and when $\gamma=1$ the failure rate of working components is $\lambda_{k}=\lambda_{1} / k$. For the presented model, Eq. (9) is transformed into Eq. (10):

$$
\lambda_{i z_{i} k_{i}}=\frac{k_{i}-\gamma\left(k_{i}-1\right)}{k_{i}} \lambda_{i z_{i} 1} .
$$

We combined Eqs. (8) and (10), so, the reliability of the systems with active redundancy strategy, and the failure rate depends on the number of working elements and can be calculated in Eq. (11) as shown in Box I.

$$
\begin{aligned}
R_{l_{1}}(t) & =\sum_{i=k_{i}}^{n_{i}} P_{i}(t)=\left(\prod_{j=k_{i}}^{n_{i}} \frac{j-\gamma(j-1)}{j} \lambda_{i z_{i} 1}\right) \\
& \times \sum_{i=k_{i}}^{n_{i}}\left[\frac{n_{i} !}{i\left(k_{i}-1\right) !}\left(\prod_{\substack{\omega=k_{i} \\
\omega \neq i}}^{n_{i}} \frac{1}{\{\{\omega-\gamma(\omega-1)\}-\{i-\gamma(i-1)\}\} \lambda_{i z_{i} 1}}\right) \times \frac{e^{-\{i-\gamma(i-1)\} \lambda_{i z_{i} 1} t}}{\{i-\gamma(i-1)\} \lambda_{i z_{i} 1} 1}\right] .
\end{aligned}
$$


The reliability formula for a cold standby subsystem is presented in Eq. (12); in these subsystems, the switch detects the failures of a working component and changes the failed component with a new one. The switching system is a discrete detection switching and may work in each detection by the probability $p$ and maybe failed in each detection by the probability $(1-p)$.

$$
\begin{aligned}
& R_{l_{2}}(t)=\sum_{j=0}^{n_{i}-k_{i}-1} \\
& \quad\left\{(1-p) p^{j} \sum_{m=0}^{j} \frac{e^{-k_{i} \lambda_{i z_{i} k_{i}} t} \cdot\left(k_{i} \lambda_{i z_{i} k_{i}} t\right)^{m}}{m !}\right\} \\
& +p^{n_{i}-k_{i}} \sum_{m=0}^{n_{i}-k_{i}} \frac{e^{-k_{i} \lambda_{i z_{i} k_{i}} t} \cdot\left(k_{i} \lambda_{i z_{i} k_{i}} t\right)^{m}}{m !} .
\end{aligned}
$$

Since RAP belongs to NP-hard problems and the first objective function of this problem is nonlinear, the exact solutions have less efficiency in solving this problem. So, we used the NSGA-II and NRGA metaheuristic algorithms to solve the presented model. These algorithms are presented in the next section.

\section{Solving methods}

We used NSGA-II and NRGA algorithms to solve the presented problem. These two algorithms are based on population, so the solution structures of both algorithms are the same.

\subsection{Solution encoding}

Each solution is a $4 \times s$ matrix. The $i$ th column of the matrix belongs to the $i$ th subsystem. The first row of the matrix represents the redundancy strategy of the subsystem components; the second row shows the component type of the subsystem. The third part contains the number of components in each subsystem, and the last row is the failure rate reduction coefficient of the component in the subsystem. The structure of an encoded solution is shown in Figure 1. In this figure, in the first subsystem, 4 components of type three exist, and the components have an active redundancy strategy. Also, the failure rate of the components in this subsystem was reduced by $18.66 \%$.

\subsection{NSGA-II algorithm}

In 1994, Srinivas and Deb [63] used Goldberg ideas and presented the concepts of NSGA. This algorithm is efficient but too complicated, unable to solve multiobjective problems. Deb et al. [64] presented the NSGA-II algorithm to overcome the weakness of the NSGA algorithm regarding particle election and the complexity. In this algorithm, the Pareto solutions are obtained using dominant and non-dominant solutions, and the mutation operator of the genetic algorithm is used to find the new solutions.

\subsection{NRGA algorithm}

Improvement of operators is a way to improve the efficiency of multi-objective algorithms. Improvement of selection operators has more effects on the improvement of algorithms efficiency and makes the evolutionary algorithms more converging. So, Al Jadaan et al. [65] improved an evolutionary multiobjective algorithm based on Ranked based roulette wheel selection and Pareto-based population ranking and called it NRGA. In this combination, a two-layer ranking is presented based on roulette wheel selection that randomly selects the new generation from old ones based on selecting the best solutions (based on fitness and span). The NRGA can better achieve a wide range of solutions and converge to optimal Pareto versus other evolutionary algorithms.

In both algorithms, the solutions in each population rank are based on their non-dominant rate. The solutions in the first category are the best nondominant solutions, and the solutions in the last category are the worst non-dominant solutions. So, the solutions in the first category have the maximum fitting, and the solutions in the last category have the minimum fitting. After ranking the categories, the solutions in each category rank are based on the swarm. The solution with maximum swarm has the maximum rank, and the solution with minimum swarm has the

\begin{tabular}{|c|c|c|c|c|c|c|c|c|c|c|c|c|c|c|}
\hline & \multicolumn{14}{|c|}{ Subsystem } \\
\hline & 1 & 2 & 3 & 4 & 5 & 6 & 7 & 8 & 9 & 10 & 11 & 12 & 13 & 14 \\
\hline Redundancy Strategy & A & $\mathrm{S}$ & $\mathrm{S}$ & A & A & S & A & S & $\mathrm{A}$ & A & $\mathrm{S}$ & S & A & S \\
\hline Components Type & 2 & 1 & 3 & 2 & 3 & 4 & 3 & 1 & 4 & 2 & 3 & 2 & 1 & 2 \\
\hline Components Number & 4 & 2 & 3 & 4 & 1 & 1 & 3 & 5 & 3 & 2 & 6 & 4 & 2 & 1 \\
\hline Coefficients of Decreasing Failure Rates & 0.1866 & 0.0134 & 0.1642 & 0.0527 & 0.073 & 0.1957 & 0.1921 & 0.0381 & 0.1931 & 0.1312 & 0.1923 & 0.1968 & 0.1205 & 0.079 \\
\hline
\end{tabular}
minimum rank in the category. NSGA-II and NRGA differ in selecting a strategy, ordering the population,

Figure 1. Encoded solution. 
and selecting for the next generation. NRGA used RRWS instead of the tournament operator. In this operator, the solutions with better fitting have a higher chance to be selected for reproduction and creation of the next generation. Al Jadaan et al. [65] used a selected modified algorithm based on the roulette wheel in which each solution has the fitting value equal to the rank of the solution in the population. The solutions in the population rank are based on two specifications. First, the rank of the containing category of the solution, and second the rank of the solution in the category. For selecting a solution, at first, a non-dominant category must be selected. The probability of selecting the $i$ th non-dominant category is calculated using Eq. (13) [65]:

$$
p_{i}=\frac{2 \times \operatorname{rank}_{i}}{N_{f} \times\left(N_{f}+1\right)}=\frac{\operatorname{rank}_{i}}{\sum_{i=1}^{p} \operatorname{rank}_{i}},
$$

where $\operatorname{rank}_{i}$ is the rank of $i$ th category and $N_{f}$ is the number of the categories. The probability of selecting $j$ th solution in $i$ th non-dominant category is calculated using Eq. (14) [65]:

$$
p_{j i}=\frac{2 \times \operatorname{rank}_{j i}}{N_{j} \times\left(N_{j}+1\right)}=\frac{\operatorname{rank}_{j i}}{\sum_{j=1}^{p} \operatorname{rank}_{j i}},
$$

where $N_{j}$ is the number of solutions in $i$ th category and rank $_{j i}$ is the rank of $j$ th solution in $i$ th category.

In the roulette wheel, the first two real intervals $\left[0, S_{1}\right]$ and $\left[0, S_{2}\right]$ values $S_{1}=\sum_{i=1}^{n} p_{i}$ and $S_{2}=$ $\sum_{j=1}^{m} p_{j}$ are defined. Then the solutions in each category occupy a certain amount of $\left[0, S_{1}\right]$ and $\left[0, S_{2}\right]$ based on the probability of their selection. Then two random numbers are selected between zero and one, and the first random number is used to select in $\left[0, S_{1}\right]$ and the second random number is used to select an answer in $\left[0, S_{2}\right]$. The flow chart of both algorithms is presented in Figure 2.

\subsection{Comparison metrics}

Convergence with Pareto optimal solutions and providing density and diversity among the set of solutions are two distinct and somewhat conflicting objectives in multi-objective evolutionary algorithms, a criterion that can be used alone and does not exist in absolute terms for calculating the performance of the algorithm [66].

For this reason, we used five performance metrics to better evaluate the performance of the two presented algorithms better.

\subsubsection{Maximum spread or diversity}

This index measures the length of the space cubic diameter used in the final values of the targets for the nondominant solutions. Eq. (15) shows the computational

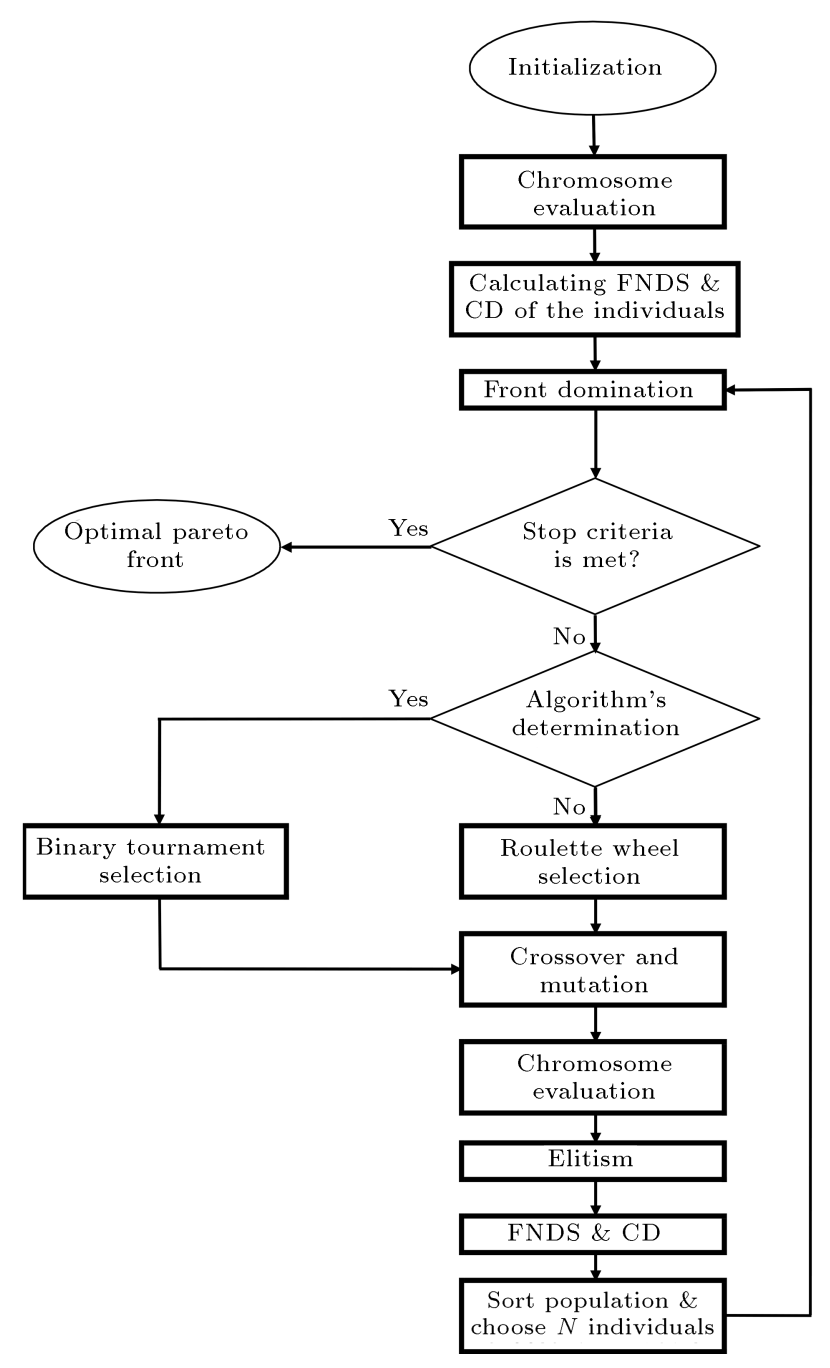

Figure 2. The results for Non-dominated Sorting Genetic Algorithm II (NSGA-II) and Non-dominated Ranked Genetic Algorithm (NRGA) algorithms.

procedure of this index. Therefore, the larger this criterion, the more the archived Pareto front spreads.

$$
D=\sqrt{\sum_{j=1}^{m}\left(\max _{i} f_{i}^{j}-\min _{i} f_{i}^{j}\right)^{2}} .
$$

4.4.2. Spacing

This scale calculates the relative distance of consecutive solutions using Eq. (16):

$$
S=\sqrt{\frac{1}{|n|} \sum_{i=1}^{n}\left(d_{i}-\bar{d}\right)^{2}},
$$

where $\bar{d}=\sum_{i=1}^{n} d_{i} /|n|$ and $d_{i}=\min _{k \in n k \neq 1} \sum_{m=1}^{2} \mid f_{m}^{i}-$ $f_{m}^{k} \mid$. This scale measures the standard deviations of different values $d_{i}$. When the solutions are close to the gathering, $S$ has smaller a value, and the performance of the algorithm with a small spacing scale is better than other algorithms [66]. 
Table 2. Data for numerical example.

\begin{tabular}{|c|c|c|c|c|c|c|c|c|c|c|c|c|c|}
\hline \multicolumn{2}{|c|}{ Subsystem } & \multicolumn{3}{|c|}{ Component type 1} & \multicolumn{3}{|c|}{ Component type 2} & \multicolumn{3}{|c|}{ Component type 3} & \multicolumn{3}{|c|}{ Component type 4} \\
\hline$i$ & $k_{i}$ & $\lambda_{i 1}$ & $\lambda_{c_{i 1}}$ & $w_{i 1}$ & $\lambda_{i 2}$ & $\lambda c_{i 2}$ & $w_{i 2}$ & $\lambda_{i 3}$ & $\lambda c_{i 3}$ & $w_{i 3}$ & $\lambda_{i 4}$ & $\lambda c_{i 4}$ & $w_{i 4}$ \\
\hline 1 & 1 & 0.001054 & 1 & 3 & 0.000726 & 1 & 4 & 0.000943 & 2 & 2 & 0.000513 & 2 & 5 \\
\hline 2 & 2 & 0.000513 & 2 & 8 & 0.000619 & 1 & 10 & 0.000726 & 1 & 9 & & - & \\
\hline 3 & 1 & 0.001625 & 2 & 7 & 0.001054 & 3 & 5 & 0.001393 & 1 & 6 & 0.000834 & 4 & 4 \\
\hline 4 & 2 & 0.001863 & 3 & 5 & 0.001393 & 4 & 6 & 0.001625 & 5 & 4 & & - & \\
\hline 5 & 1 & 0.000619 & 2 & 4 & 0.000726 & 2 & 3 & 0.000513 & 3 & 5 & & - & \\
\hline 6 & 2 & 0.000101 & 3 & 5 & 0.000202 & 3 & 4 & 0.000305 & 2 & 5 & 0.000408 & 2 & 4 \\
\hline 7 & 1 & 0.000943 & 4 & 7 & 0.000834 & 4 & 8 & 0.000619 & 5 & 9 & & - & \\
\hline 8 & 2 & 0.002107 & 3 & 4 & 0.001054 & 5 & 7 & 0.000943 & 6 & 6 & & - & \\
\hline 9 & 3 & 0.000305 & 2 & 8 & 0.000101 & 3 & 9 & 0.000408 & 4 & 7 & 0.000943 & 3 & 8 \\
\hline 10 & 3 & 0.001863 & 4 & 6 & 0.001625 & 4 & 5 & 0.001054 & 5 & 6 & & - & \\
\hline 11 & 3 & 0.000619 & 3 & 5 & 0.000513 & 4 & 6 & 0.000408 & 5 & 6 & & - & \\
\hline 12 & 1 & 0.002357 & 2 & 4 & 0.001985 & 3 & 5 & 0.001625 & 4 & 6 & 0.001054 & 5 & 7 \\
\hline 13 & 2 & 0.000202 & 2 & 5 & 0.000101 & 3 & 5 & 0.000305 & 2 & 6 & & - & \\
\hline 14 & 3 & 0.001054 & 4 & 6 & 0.000834 & 4 & 7 & 0.000513 & 5 & 6 & 0.000101 & 6 & 9 \\
\hline
\end{tabular}

\subsubsection{Number of Pareto Solution (NPS)}

This scale shows the NPSs in each algorithm.

\subsubsection{Mean Ideal Distance (MID)}

This scale indicates the distance to the ideal Pareto level and is calculated using Eq. (17). The lower values of this scale indicate that the algorithm is working properly.

$$
M I D=\frac{\sum_{i=1}^{n} \sqrt{f_{i 1}^{2}+f_{i 2}^{2}}}{n},
$$

where $f_{i 1}$ and $f_{i 2}$ are the first and second objective functions in $i$ th solution.

\subsubsection{Time of algorithm}

This scale defines the time of the algorithm running to satisfy stop criteria.

\section{Numerical example}

In this section, we present a numerical example to illustrate the effectiveness of the presented algorithms. The example data are obtained from the data of Coit and Smith [5]. The example is a series-parallel system with the k-out-of-n subsystem. Three or four different component types are available for each subsystem, and the redundancy strategy of each subsystem is a variable. The cost, weight, and failure rate of components and a minimum number of components in each subsystem are presented in Table 2. The maximum number of each subsystem component is six. The objectives are maximizing system reliability and minimizing system cost underweight constraint [6].
Table 3. Range of algorithms tuned parameters.

\begin{tabular}{ccc}
\hline Solving methodologies & Parameter & Range \\
\hline \multirow{2}{*}{ NSGA-II } & $n P_{o p}$ & $50-100$ \\
& $P_{c}$ & $0.3-0.6$ \\
& $P_{m_{1}}$ & $0.1-0.3$ \\
& $P_{m_{2}}$ & $0.1-0.3$ \\
& & \\
NRGA & Pop & $50-100$ \\
& $P_{c}$ & $0.3-0.6$ \\
& $P_{m_{1}}$ & $0.1-0.3$ \\
& $P_{m_{2}}$ & $0.1-0.3$ \\
\hline
\end{tabular}

Also, the switch reliability is considered as $p=0.99$ [19]. The internal connection cost for all subsystems is $\theta_{i z_{i}}=0.25$ [62] and $\gamma=0.2$, also the cost of reducing components failure rate is $0.75 C_{i z_{i}}$.

\subsection{Parameter tuning}

The parameters of NRGA and NSGA-II algorithms are tuned in this section. The Response Surface Methodology (RSM) is used for parameter tuning. These parameters are population size $(n P o p)$, crossover rate $\left(P_{c}\right)$, mutation rate $\left(P_{m_{1}}\right)$, and max-min operator $\left(P_{m_{2}}\right)$. Table 3 presents the range of these parameters and Table 4 shows the results of parameter tuning.

\subsection{Results}

This section deals with comparing the results of NSGAII and NRGA algorithms. For this comparison, we used a Laptop with 6G RAM and $1.73 \mathrm{GH}$ CPU 
Table 4. Optimal values of parameters.

\begin{tabular}{ccc}
\hline Solving methodologies & Parameter & Optimum value \\
\hline \multirow{2}{*}{ NSGA-II } & $P_{c}$ & 79 \\
& $P_{m_{1}}$ & 0.30 \\
& $P_{m_{2}}$ & 0.30 \\
& $n P_{o p}$ & 0.10 \\
NRGA & $P_{c}$ & 0.45 \\
& $P_{m_{1}}$ & 0.20 \\
& $P_{m_{2}}$ & 0.20 \\
\hline
\end{tabular}

speed, and the algorithm codded using MATLAB 2018. Each algorithm ran five times with the optimal values of parameters. The iteration of each algorithm was considered 100. The results of the algorithm performance were presented in Figure 3. In NPS, concerning diversity and time scales, the NSGA-II has a better performance than those in the NRGA algorithm, and in other scales, NRGA is better than NSGA-II.

\subsubsection{Results analysis}

To find the difference between the results of the indexes, we used a one-way ANOVA test using $1-\alpha=$ 0.95. Table 5 presents the results for the ANOVA test.

The ANOVA test results show that there are meaningful differences between the indexes of the two algorithms, and reject the assumption that the results of the two algorithms are the same.

\subsubsection{Sensitivity analysis}

To further evaluate the model in this section, we intend to solve 33 different numerical examples for the presented multi-objective model using NSGA-II and NRGA algorithms. The information for these 33 numerical examples is similar to Table 2. However, the available weight of the system varies from 159 to 191. The result of the performance indices of each algorithm on these 33 numerical examples are graphically illustrated in Figure 4.

\section{Managerial insights}

Increasing the number of parallel components in a system can promote the reliability of this system, but it is not sufficient. Related researches show that
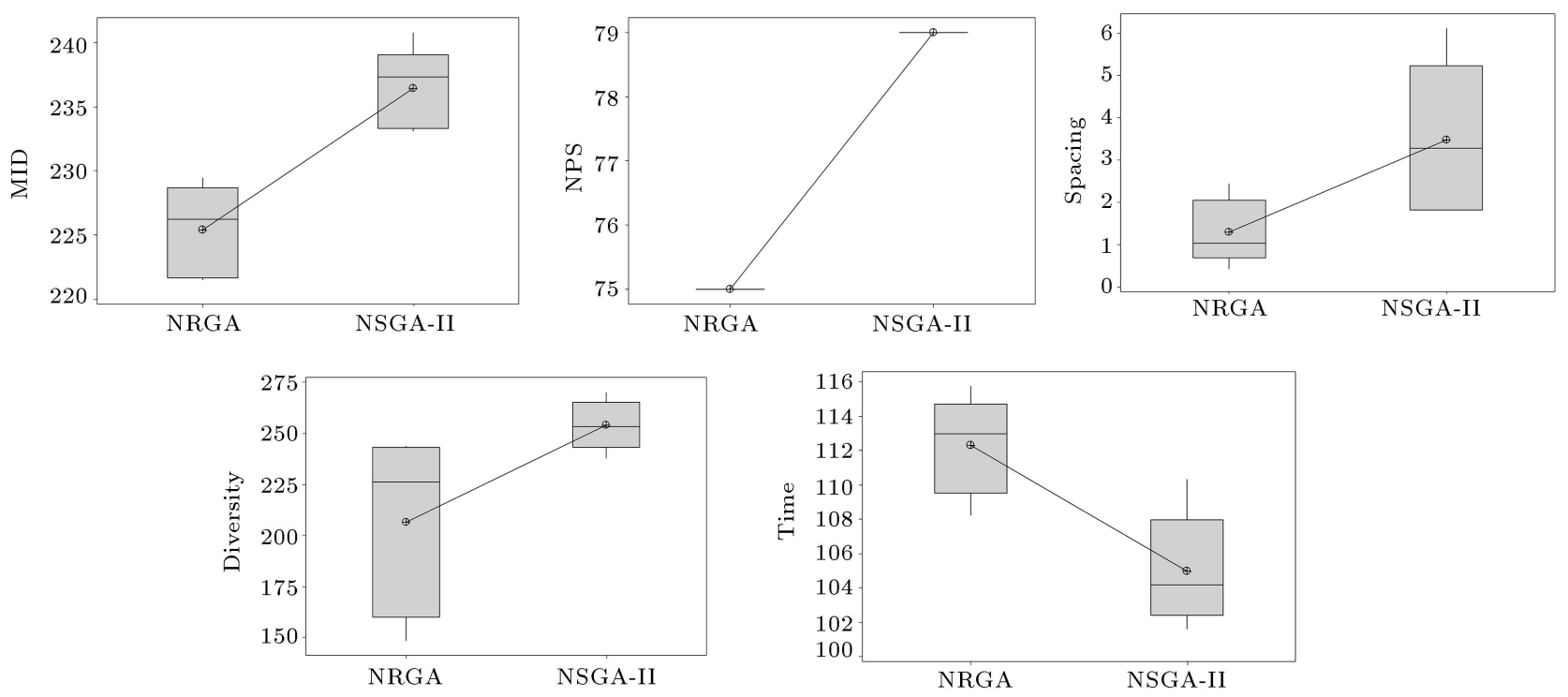

Figure 3. The results for Non-dominated Sorting Genetic Algorithm II (NSGA-II) and Non-dominated Ranked Genetic Algorithm (NRGA) algorithms.

Table 5. Results of algorithms.

\begin{tabular}{cccc}
\hline Performance metrics & $\boldsymbol{P}$-value & Result & Final result \\
\hline MID & 0.001 & H0 is rejected & NRGA \\
NPS & 0.000 & H0 is rejected & NSGA-II \\
Spacing & 0.039 & H0 is rejected & NSGA-II \\
Diversity & 0.047 & H0 is rejected & NRGA \\
Time & 0.006 & H0 is rejected & NSGA-II \\
\hline
\end{tabular}


MID

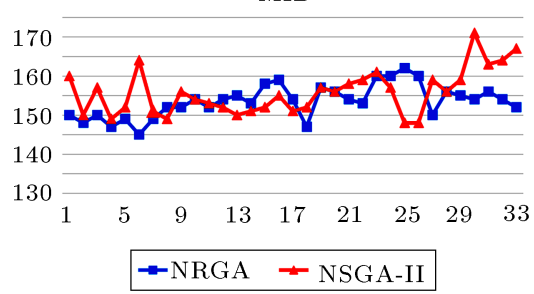

NPS

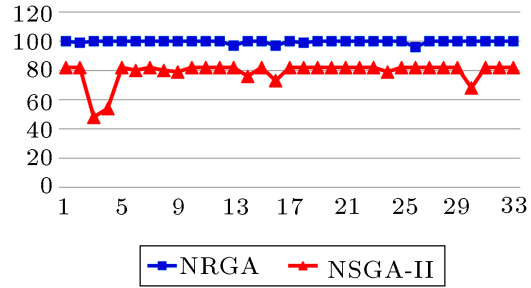

Spacing

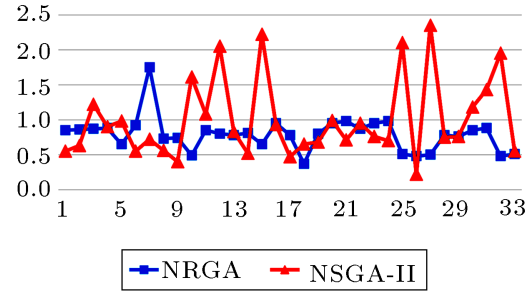

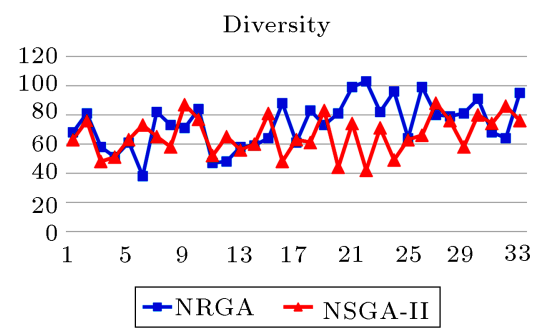

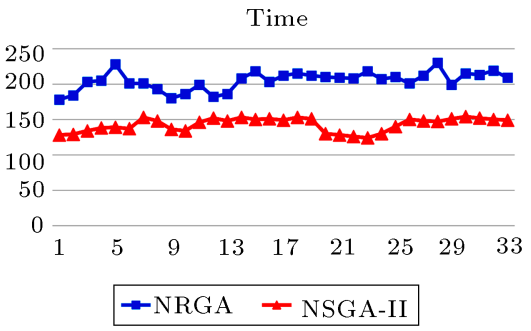

Figure 4. Sensitivity analysis of the proposed model by Non-dominated Sorting Genetic Algorithm II (NSGA-II) and Non-dominated Ranked Genetic Algorithm (NRGA) algorithms.

improving the performance of each used component in a system can be utilized as another way to improve the reliability of the entire system. Therefore, in this paper, both of these criteria are employed to improve the performance of the system. It should be considered that the required parameters for designing the problem are formed based on the effects of each approach mentioned above and their related costs. On the other hand, if there is a budget capacity constraint, effort in promoting components' availability leads to a less redundancy assignment and vice versa.

Many realistic examples of failure rate improvement are given in the literature. For instance, consider the action of the installation of a vibration monitoring system for the FD fan and the ID fan. Vibration monitoring can monitor the health condition of the fans, and preventive replacements can be performed to prevent unexpected failures. Therefore, the failure rates of the can be reduced, thereby reducing the failure rate of the generating unit from the $300 \mathrm{MW}$ state to the $150 \mathrm{MW}$ state. Before adopting the vibration monitoring system, the reduction in the failure rates of the fans, i.e., the benefit of installing the vibration monitoring system, can be estimated based on the failure histories, or by the vibration monitoring system provider based on their experiences. Other examples are: installing monitor systems and maintenance planning.

\section{Conclusion and further studies}

Many parameters affect the reliability of the systems, and the failure rate of components is one of the most important parameters. In this paper, we worked on a two-objective reliability model. In this model, the failure rate of the components is constant and depends on the number of working components in the system. The components failure rates could be cost-effective. The system contains $s$ subsystems, and the subsystems have the k-out-of-n configuration. All subsystems may have two active and cold standby redundancy strategies that are system variables. Besides, the number and the type of each subsystem component and the reduction of the failure of the component rate are other variables of the model. Because Redundancy Allocation Problem (RAP) belongs to the NP-hard problem, we used Non-dominated Sorting Genetic Algorithm II (NSGAII) and Non-dominated Ranked Genetic Algorithm (NRGA) multi-objective algorithms for solving the presented problem. Also, we used 5 different indexes for comparing the algorithm performance. The results showed that NSGA-II has better performance in Number of Pareto Solution (NPS), diversity, and time indexes, and for other indexes, the NRGA has better performance.

For further studies, the components can be considered as repairable components. In addition, the combination of different components in each subsystem can also be considered a new idea. Also, another multiobjective metaheuristic algorithm can be considered for solving the proposed problem.

\section{References}

1. Fyffe, D.E., Hines, W.W., and Lee, N.K. "System reliability allocation and a computational algorithm", IEEE Transactions on Reliability, 17(2), pp. 64-69 (1968).

2. Chern, M.S. "On the computational complexity of re- 
liability redundancy allocation in a series system", $O p$ erations Research Letters, 11(5), pp. 309-315 (1992).

3. Misra, K.B. and Sharma, U. "Multi-criteria optimization for combined reliability and redundancy allocation in systems employing mixed redundancies", Microelectronics Reliability, 31(2-3), pp. 323-335 (1991).

4. Ida, K. "System reliability optimization with several failure modes by genetic algorithm", In Proceeding of 16th International Conference on Computers \& Industrial Engineering, pp. 349-352 (1994).

5. Coit, D.W. and Smith, A.E. "Optimization approaches to the redundancy allocation problem for seriesparallel systems", In Fourth Industrial Engineering Research Conference Proceedings, pp. 342-349 (1995).

6. Coit, D.W. and Liu, J.C. "System reliability optimization with k-out-of-n subsystems", International Journal of Reliability, Quality and Safety Engineering, 7(02), pp. 129-142 (2000).

7. Hsieh, Y.C. and You, P.S. "An effective immune based two-phase approach for the optimal reliabilityredundancy allocation problem", Applied Mathematics and Computation, 218(4), pp. 1297-1307 (2011).

8. Hsieh, T.J. and Yeh, W.C. "Penalty guided bees search for redundancy allocation problems with a mix of components in series-parallel systems", Computers \& Operations Research, 39(11), pp. 2688-2704 (2012).

9. Busacca, P.G., Marseguerra, M., and Zio, E. "Multiobjective optimization by genetic algorithms: application to safety systems", Reliability Engineering \& System Safety, 72(1), pp. 59-74 (2001).

10. Coit, D.W. and Jin, T. "Multi-criteria optimization: Maximization of a system reliability estimate and minimization of the estimate variance", In Proceedings of the 2001 European Safety \& Reliability International Conf. (ESREL), Turin, Italy (2001).

11. Baheranwala, F., Coit, D.W., and Kulturel-Konak, S. "Solution of stochastic multi-objective system design problems", In IIE Annual Conference. Proceedings (2004).

12. Salazar, D., Rocco, C.M., and Galván, B.J. "Optimization of constrained multiple-objective reliability problems using evolutionary algorithms", Reliability Engineering \& System Safety, 91(9), pp. 1057-1070 (2006).

13. Kulturel-Konak, S., Coit, D.W., and Baheranwala, F. "Pruned Pareto-optimal sets for the system redundancy allocation problem based on multiple prioritized objectives", Journal of Heuristics, 14(4), p. 335 (2008).

14. Taboada, H.A., Baheranwala, F., Coit, D.W., and Wattanapongsakorn, N. "Practical solutions for multiobjective optimization: An application to system reliability design problems", Reliability Engineering \& System Safety, 92(3), pp. 314-322 (2007).

15. Taboada, H.A. and Coit, D.W. "Development of a new multiple objective prioritized genetic algorithm", In IIE Annual Conference. Proceedings, p. 176 (2008).
16. Liang, Y.C. and Lo, M.H. "Multi-objective redundancy allocation optimization using a variable neighborhood search algorithm", Journal of Heuristics, 16(3), pp. 511-535 (2010).

17. Soylu, B. and Ulusoy, S.K. "A preference ordered classification for a multi-objective max-min redundancy allocation problem", Computers \& Operations Research, 38(12), pp. 1855-1866 (2011).

18. Coit, D.W. "Cold-standby redundancy optimization for non-repairable systems", Iie Transactions, 33(6), pp. $471-478$ (2001).

19. Coit, D.W. "Maximization of system reliability with a choice of redundancy strategies", IIE Transactions, 35(6), pp. 535-543 (2003).

20. Tavakkoli-Moghaddam, R., Safari, J., and Sassani, F. "Reliability optimization of series-parallel systems with a choice of redundancy strategies using a genetic algorithm", Reliability Engineering \& System Safety, 93(4), pp. 550-556 (2008).

21. Safari, J. and Tavakkoli-Moghaddam, R. "A redundancy allocation problem with the choice of redundancy strategies by a memetic algorithm", Journal of Industrial Engineering, International, 6(11), pp. 6-16 (2010).

22. Amari, S.V. "Reliability of k-out-of-n standby systems with gamma distributions", In 2012 Proceedings Annual Reliability and Maintainability Symposium, pp. 1-6 (2012).

23. Dhingra, A.K. "Optimal apportionment of reliability and redundancy in series systems under multiple objectives", IEEE Transactions on Reliability, 41(4), pp. 576-582 (1992).

24. Ghafarian Salehi Nezhad, A., Eshraghniaye Jahromi, A., Salmani, M.H., and Ghasemi, F. "A four-phase algorithm to improve reliability in series-parallel systems with redundancy allocation", Scientia Iranica, 21(3), pp. 1072-1082 (2014).

25. Azaron, A., Katagiri, H., Kato, K., and Sakawa, M. "Reliability evaluation and optimization of dissimilarcomponent cold-standby redundant systems", Journal of the Operations Research Society of Japan, 48(1), pp. 71-88 (2005).

26. Azaron, A., Perkgoz, C., Katagiri, H., Kato, K., and Sakawa, M. "Multi-objective reliability optimization for dissimilar-unit cold-standby systems using a genetic algorithm", Computers \& Operations Research, 36(5), pp. 1562-1571 (2009).

27. Ebrahim Nezhad, M., Maleki, V.B., Pasandideh, H.R., and Safari, J. "Increasing the reliability and the profit in a redundancy allocation problem", International Journal of Applied Operational Research, 1(2), pp. 5764 (2011).

28. Chambari, A., Najafi, A.A., Rahmati, S.H.A., and Karimi, A. "An efficient simulated annealing algorithm for the redundancy allocation problem with a choice of redundancy strategies", Reliability Engineering \& System Safety, 119, pp. 158-164 (2013). 
29. Azimi, P., Hemmati, M., and Chambari, A. "Solving the redundancy allocation problem of k-out-of-n with non-exponential repairable components using optimization via simulation approach", Scientia Iranica, 24(3), pp. 1547-1560 (2017).

30. Pourkarim Guilani, P., Zaretalab, A., and Niaki, S.T.A. "A bi-objective model to optimize reliability and cost of k-out-of-n series-parallel systems with tristate components", Scientia Iranica, Transactions E, Industrial Engineering, 24(3), pp. 1585-1602 (2017).

31. Sharifi, M., Memariani, A., and Noorossana, R. "Real time study of a k-out-of-n system: $n$ identical elements with constant fuzzy failure", World Applied Sciences Journal, 8(9), pp. 1136-1143 (2010).

32. Sharifi, M., Ganjian, M., and Ghajar, H.R. "Expansion of reliability models based on Markov chain with consideration of fuzzy failure rates: System with two parallel and identical elements with constant failure rates", In IEEE International Conference on Computational Intelligence for Modelling, Control and Automation and International Conference on Intelligent Agents, Web Technologies and Internet Commerce (CIMCA-IAWTIC'06), 2, pp. 1022-1026 (2005).

33. Lins, I.D. and Droguett, E.L. "Redundancy allocation problems considering systems with imperfect repairs using multi-objective genetic algorithms and discrete event simulation", Simulation Modelling Practice and Theory, 19(1), pp. 362-381 (2011).

34. Ouzineb, M., Nourelfath, M., and Gendreau, M. "Tabu search for the redundancy allocation problem of homogenous series-parallel multi-state systems", Reliability Engineering \& System Safety, 93(8), pp. 1257-1272 (2008).

35. Sharma, V.K. and Agarwal, M. "Ant colony optimization approach to heterogeneous redundancy in multistate systems with multi-state components", In 8th International IEEE Conference on Reliability, Maintainability and Safety, pp. 116-121 (2009).

36. Lins, I.D. and Droguett, E.L. "Multi-objective optimization of availability and cost in repairable systems design via genetic algorithms and discrete event simulation", Pesquisa Operacional, 29(1), pp. 43-66 (2009).

37. Ouzineb, M., Nourelfath, M., and Gendreau, M. "A heuristic method for non-homogeneous redundancy optimization of series-parallel multi-state systems", Journal of Heuristics, 17(1), pp. 1-22 (2011).

38. Ebrahimipour, V. and Sheikhalishahi, M. "Application of multi-objective particle swarm optimization to solve a fuzzy multi-objective reliability redundancy allocation problem", In IEEE International Systems Conference, pp. 326-333 (2011).

39. Lins, I.D. and Droguett, E.L. "Redundancy allocation problems considering systems with imperfect repairs using multi-objective genetic algorithms and discrete event simulation", Simulation Modelling Practice and Theory, 19(1), pp. 362-381 (2011).
40. Garg, H. and Sharma, S.P. "Multi-objective reliabilityredundancy allocation problem using particle swarm optimization", Computers \& Industrial Engineering, 64(1), pp. 247-255 (2013).

41. Garg, H., Rani, M., and Sharma, S.P. "An efficient two phase approach for solving reliability-redundancy allocation problem using artificial bee colony technique", Computers \& Operations Research, 40(12), pp. 29612969 (2013).

42. Levitin, G., Xing, L., Ben-Haim, H., and Dai, Y. "Reliability of series-parallel systems with random failure propagation time", IEEE Transactions on Reliability, 62(3), pp. 637-647 (2013).

43. Maatouk, I., Châtelet, E., and Chebbo, N. "Availability maximization and cost study in multi-state systems", In IEEE Proceedings Annual Reliability and Maintainability Symposium (RAMS), pp. 1-6 (2013).

44. Gago, J., Hartillo, I., Puerto, J., and Ucha, J.M. "Exact cost minimization of a series-parallel reliable system with multiple component choices using an algebraic method", Computers \& Operations Research, 40(11), pp. 2752-2759 (2013).

45. Ebrahimipour, V., Asadzadeh, S.M., and Azadeh, A. "An emotional learning-based fuzzy inference system for improvement of system reliability evaluation in redundancy allocation problem", The International Journal of Advanced Manufacturing Technology, 66(912), pp. 1657-1672 (2013).

46. Liu, Y., Huang, H.Z., Wang, Z., Li, Y., and Yang, $Y$. "A joint redundancy and imperfect maintenance strategy optimization for multi-state systems", IEEE Transactions on Reliability, 62(2), pp. 368-378 (2013).

47. Khalili-Damghani, K., Abtahi, A.R., and Tavana, M. "A decision support system for solving multi-objective redundancy allocation problems", Quality and Reliability Engineering International, 30(8), pp. 1249-1262 (2014).

48. Guilani, P.P., Sharifi, M., Niaki, S.T.A., and Zaretalab, A. "Reliability evaluation of non-reparable threestate systems using Markov model and its comparison with the UGF and the recursive methods", Reliability Engineering \& System Safety, 129, pp. 29-35 (2014).

49. Sharifi, M., Cheragh, G., Maljaii, K.D., Zaretalab, A., Daei, F., and Vahid, A. "Reliability optimization of a series-parallel k-out-of-n system with failure rates depends on working component of system", International Journal of Industrial Engineering, 22(4), pp. 438-453 (2015).

50. Mousavi, S.M., Alikar, N., Niaki, S.T.A., and Bahreininejad, A. "Two tuned multi-objective metaheuristic algorithms for solving a fuzzy multi-state redundancy allocation problem under discount strategies", Applied Mathematical Modelling, 39(22), pp. 6968-6989 (2015).

51. Zaretalab, A., Hajipour, V., Sharifi, M., and Shahriari, M.R. "A knowledge-based archive multi-objective simulated annealing algorithm to optimize series-parallel 
system with choice of redundancy strategies", Computers \& Industrial Engineering, 80, pp. 33-44 (2015).

52. Miriha, M., Niaki, S.T.A., Karimi, B., and Zaretalab, A. "Bi-objective reliability optimization of switchmode k-out-of-n series-parallel systems with active and cold standby components having failure rates dependent on the number of components", Arabian Journal for Science and Engineering, 42(12), pp. 5305-5320 (2017).

53. Pourkarim Guilani, P., Azimi, P., Sharifi, M., and Amiri, M. "Redundancy allocation problem with a mixed strategy for a system with k-out-of-n subsystems and time-dependent failure rates based on Weibull distribution: An optimization via simulation approach", Scientia Iranica, 26(2), pp. 1023-1038 (2019).

54. Sharifi, M., Saadvandi, M., and Shahriari, M.R. "Presenting a series-parallel redundancy allocation problem with multi-state components using recursive algorithm and meta-heuristic", Scientia Iranica, 27(2), pp. 970982 (2020).

55. Zaretalab, A. and Hajipour, V. "An extended simulated annealing based on the memory structure to solve redundancy allocation problem", Journal of Advanced Manufacturing Systems, 18(04), pp. 527-548 (2019).

56. Sharifi, M., Shahriari, M.R., and Zaretalab, A. "The effects of technical and organizational activities on redundancy allocation problem with choice of selecting redundancy strategies using the memetic algorithm", International Journal of Industrial Mathematics, 11(3), pp. 165-176 (2019).

57. Sharifi, M. and Khoshniat, S. "Optimization the availability of a system with short circuit and common cause failures", International Journal of Industrial Mathematics, 11(4), pp. 239-248 (2019).

58. Sharifi, M., Moghaddam, T.A., and Shahriari, M. "Multi-objective redundancy allocation problem with weighted-k-out-of-n subsystems", Heliyon, 5(12), e02346 (2019)

59. Alamdari, A.B. and Sharifi, M. "Solving a joint availability-redundancy optimization model with multistate components and metaheuristic approach", International Journal of Industrial Mathematics, 12(1), pp. 59-70 (2020).

60. Zaretalab, A., Hajipour, V., and Tavana, M. "Redundancy allocation problem with multi-state component systems and reliable supplier selection", Reliability Engineering \& System Safety, 193, p. 106629 (2020).

61. Sharifi, M. and Taghipour, S. "Optimizing a redundancy allocation problem with open-circuit and shortcircuit failure modes in components and subsystems level", Journal of Engineering Optimization (In press).

62. Wang, Z., Chen, T., Tang, K., and Yao, X. "A multiobjective approach to redundancy allocation problem in parallel-series systems", In 2009 IEEE Congress on Evolutionary Computation, pp. 582-589 (2009).
63. Srinivas, N. and Deb, K. "Multi-objective optimization using non-dominated sorting in genetic algorithms", Evolutionary Computation, 2(3), pp. 221-248 (1994).

64. Deb, K., Agrawal, S., Pratap, A., and Meyarivan, T. "A fast elitist non-dominated sorting genetic algorithm for multi-objective optimization: NSGA-II", In International Conference on Parallel Problem Solving from Nature, Springer, Berlin, Heidelberg, pp. 849-858 (2000).

65. Al Jadaan, O., Rajamani, L., and Rao, C.R. "Nondominated ranked genetic algorithm for solving multiobjective optimization problems: NRGA", Journal of Theoretical and Applied Information Technology, 4(1), pp. 60-67 (2008).

66. Deb, K., Multi-Objective Optimization Using Evolutionary Algorithms, John Wiley \& Sons, 16 (2001).

\section{Biographies}

Mani Sharifi is an Associate Professor at the Faculty of Industrial and Mechanical Engineering in Qazvin Islamic Azad University, Qazvin, Iran. Nowadays, he is a post-doctoral research fellow at Ryerson University, the Reliability, Risk, and Maintenance Research Laboratory (RRMR Lab). He holds a BSc degree from Qazvin Islamic Azad University, an MSc degree from the south Tehran branch of Islamic Azad University, and a PhD degree from Tehran Research and Science Islamic Azad University in Industrial Engineering. He was the managerial editor of the Journal of Optimization in Industrial Engineering. His area of interest includes Reliability Engineering, Combinatorial Optimization, Statistical Optimization as well as Production Scheduling.

Ghasem Cheragh obtained his MSc degree in Industrial Engineering from Islamic Azad University, Qazvin Branch (QIAU) in Iran. He received his BSc degree in Industrial Engineering from Islamic Azad University, Karaj Branch (KIAU). His research interests are Reliability Engineering, Quality management systems, and Facility optimization. He has published a number of papers in journals such as. International Journal of Industrial Engineering Theory Applications and Practice, Sharif Scientific Research Journal (Industrial Engineering), and Industrial Management Scientific Research Journal (Allame Tabatabaei university).

Kamran Dashti Maljaii obtained his MSc degree in Industrial Engineering from Islamic Azad University, Qazvin Branch (QIAU) in Iran. He received his BSc degree in Industrial Engineering from Islamic Azad University, Karaj Branch (KIAU). His research interests are reliability engineering, quality management systems, and facility optimization. He has published a number of papers in journals such as. International 
Journal of Industrial Engineering: Theory Applications and Practice, Sharif Scientific Research Journal (Industrial Engineering), and Industrial Management Scientific Research Journal (Allame Tabatabaei University).

Arash Zaretalab obtained his PhD degree in Industrial Engineering from Amirkabir University of Technology (Tehran Polytechnic) in Iran. He received his $\mathrm{BSc}$ and $\mathrm{MSc}$ degrees in Industrial Engineering from Islamic Azad University, Qazvin Branch (QIAU). His research interests are Reliability Engineering, Combinatorial Optimization, Computational Intelligence, Machining Process Optimization, and Data Mining. He has published a number of papers in journals such as Reliability engineering \& safety system, Computer
Operation research, Computers \& Industrial Engineering, Applied soft computing, International journal of advanced manufacturing, and among others. He has also reviewed papers for these journals.

Mohammad Shahriari is an Assistant Professor at Islamic Azad University, South Tehran Branch. He holds a BSc degree from South Tehran Islamic Azad University in industrial engineering, and an MSc degree from the south Tehran branch of Islamic Azad University, and a PhD degree from Tehran Research and Science Islamic Azad University in Operations Research. His research area of interest includes DEA, combinatorial optimization, statistical optimization, and fuzzy set theory. 